\title{
The Stable Isotope Dilution Method for Measurement of Methylmalonic Acid: a Highly Accurate Approach to the Prenatal Diagnosis of Methylmalonic Acidemia
}

\author{
ARTHUR B. ZINN, DAVID G. HINE, MAURICE J. MAHONEY, AND KAY TANAKA ${ }^{(21)}$ \\ Department of Human Genetics, Yale University School of Medicine, New Haven, Connecticut, USA
}

\begin{abstract}
Summary
We report a stable isotope dilution method for the accurate measurement of methylmalonic acid (MMA) in amniotic fluid and its successful application to the prenatal diagnosis of methylmalonic acidemia. The stable isotopically-labeled analogue of MMA, $\left(2-I^{2} \mathrm{H}_{3} \mid\right.$ methyl)malonic acid, was synthesized and used as an internal standard. Samples were extracted, methylated, and analyzed by chemical ionization gas chromatography-mass spectrometry (CI-GC-MS) operated in the selected ion monitoring (SIM) mode. The analytical method is rapid $(8 \mathrm{~h})$, ultrasensitive (lower limit $\sim 10 \mathrm{ng} / \mathrm{ml}$ ), linear over three orders of magnitude, and reproducible. The concentration of MMA in amniotic fluids from normal pregnant women was $38 \pm 15 \mathrm{ng} / \mathrm{ml}$ (mean \pm 1 S.D.). This value is 20-fold less than our previous estimate by gas chromatographic analysis, indicating that interference by co-eluting materials was eliminated by use of SIM in the present method. Amniotic fluids from eight at-risk pregnancies were examined. MMA concentration in six of the pregnancies was in the normal range whereas the concentration in the remaining two pregnancies was elevated by greater than two orders of magnitude (125-250-fold). Analysis of urine from ten at-risk pregnancies showed a smaller (31/2-9-fold) but significant increase in urinary MMA excretion in two women carrying affected fetuses. A control group of normal pregnant women had a mean urinary MMA excretion of $1.7 \pm 0.5$ $\mu \mathrm{g} / \mathrm{mg}$ creatinine, in excellent agreement with previous estimates. There was concordance in prenatal diagnosis of fetal status in all cases between amniotic fluid cell studies and MMA measurements in amniotic fluid and/or maternal urine. Amniotic fluid MMA determination by the stable isotope dilution technique provides a highly accurate technique for prenatal diagnosis of methylmalonic acidemia that is complementary to amniotic fluid cell studies, and may supplant such studies in selected instances.
\end{abstract}

\section{Speculation}

The stable isotope dilution method may be extended to prenatal diagnosis of other inborn errors of metabolism in which low molecular weight metabolites accumulate in amniotic fluid. The concentrations of metabolites, which accumulate in some of these disorders, are below the sensitivity limits of currently available analytical techniques and it is thus impossible to distinguish differences that may be present in pregnancies carrying affected and unaffected fetuses. The enhanced sensitivity of the stable isotope dilution method may permit the desired discrimination.

The inherited methylmalonic acidemias are a group of metabolic disorders characterized clinically by protein intolerance, episodic vomiting lethargy, and ketoacidosis, and biochemically by the presence of large amounts of methylmalonic acid (MMA) in blood and urine. These disorders are the result of a deficiency of methylmalonyl CoA mutase (EC 5.4.99.2) activity. Methylmalonyl CoA mutase is a mitochondrial enzyme which catalyzes the conversion of $\mathrm{L}$-methylmalonyl $\mathrm{CoA}$ to succinyl $\mathrm{CoA}$, requiring 5 '-deoxyadenosylcobalamin as a cofactor (16). Detailed biochemical and genetic studies, including somatic cell complementation studies, have delineated six distinct classes in this group of disorders. These include two defects in apoenzyme production (termed the $m u t^{\circ}$ and $m u t^{-}$classes) and four distinct defects in cofactor biosynthesis (termed the $c b l A, c b l B, c b l C$, and $c b l D$ classes) (16, 19). Each of these disorders is inherited as an autosomal recessive trait and should be amenable to prenatal diagnosis $(11,16)$.

Prenatal diagnosis of methylmalonic acidemia has previously been accomplished using three approaches: (1) direct measurement of mutase activity in cultured amniotic fluid cells $(7,10) ;(2)$ measurement of the incorporation of radioactivity from $\left[1-{ }^{14} \mathrm{C}\right]$ propionate into protein presumably via tricarboxylic acid cycle intermediates by cultured amniotic fluid cells $(13,18)$; and (3) measurement of MMA in amniotic fluid and/or maternal urine $(2,7,10,12)$. The efficacy of each of these approaches has been well established in our laboratories over the past few years, but certain disadvantages with each approach have become apparent. Approaches (1) and (2) involve the analysis of relatively large numbers of cultured amniotic fluid cells and suffer from three inherent problems: (1) a delay between the time of amniocentesis and subsequent diagnosis consequent to the length of time required to grow the requisite number of cells for analysis; (2) the possibility of cell growth failure; and (3) the possibility of contamination by cells of maternal origin. The last possibility might generate a false negative result, as Buchanan et al. (5) recently observed in the prenatal diagnosis of propionic acidemia.

The various methods, which have utilized the measurement of MMA in amniotic fluid for prenatal diagnosis, have the common advantage that they are rapid, taking only a few days to perform; however, currently existing methods are not sufficiently sensitive to determine accurately the amount of MMA in amniotic fluid from normal pregnant women. Thus, it may be impossible to distinguish normal levels from mildly elevated levels when both fall below the sensitivity limit of the analytical method. The risk of misdiagnosing a mild elevation as "normal" may be increased when the amniocentesis is performed early in the pregnancy and the fetal mass is very small. With the advent of prenatal therapy for some forms of methylmalonic acidemia $(1,14)$, the availability of a reliable, rapid analysis for MMA has assumed a special importance.

We report the development of a new, highly sensitive and reproducible method for the analysis of MMA in amniotic fluid and its successful application to the prenatal diagnosis of methylmalonic acidemia. The method utilizes the stable isotope dilution technique combined with chemical ionization-gas chromatography-mass spectrometry (CI-GC-MS) operated in the selected ion monitoring (SIM) mode. 
Table 1. Methylmalonic acid concentration in amniotic fluid and urine from pregnant women

\begin{tabular}{|c|c|c|c|c|c|}
\hline \multirow[b]{2}{*}{ Subject } & \multirow[b]{2}{*}{$\begin{array}{c}\text { Mutant class of } \\
\text { proband }^{1}\end{array}$} & \multirow[b]{2}{*}{$\begin{array}{c}\text { Gestational age } \\
\text { (wk) }\end{array}$} & \multirow[b]{2}{*}{$\begin{array}{l}\text { Amniotic fluid } \\
\text { cell studies }\end{array}$} & \multicolumn{2}{|c|}{ Methylmalonic acid concentration } \\
\hline & & & & $\begin{array}{l}\text { Amniotic fluid } \\
\text { (ng/ml) }\end{array}$ & $\begin{array}{c}\text { Urine } \\
(\mu \mathrm{g} / \mathrm{mg} \text { creatinine })\end{array}$ \\
\hline Controls $(n=14)$ & Normal & $15-24(\mathrm{AF}, \mathrm{U})^{2}$ & N.D. ${ }^{4}$ & $38 \pm 15^{5}$ & $1.7 \pm 0.5^{5}$ \\
\hline \multicolumn{6}{|l|}{ At risk pregnancies } \\
\hline Case 1 & $c b l B$ & $18(\mathrm{AF})$ & + & 4840 & N.D. \\
\hline Case 2 & N.D. & $17(\mathrm{AF})$ & - & 58 & N.D. \\
\hline Case 3 & $m u t$ & $16(\mathrm{AF}, \mathrm{U})$ & + & 10600 & 15.5 \\
\hline Case 4 & $c b l A$ & $18(\mathrm{AF}, \mathrm{U})$ & - & 37 & 0.8 \\
\hline Case 5 & $c b l B$ & $20(\mathrm{AF}, \mathrm{U})$ & - & 61 & 2.4 \\
\hline Case 6 & $c b l B$ & $17(\mathrm{AF}, \mathrm{U})$ & - & 38 & 0.9 \\
\hline Case 7 & $m u t$ & $19(\mathrm{AF}, \mathrm{U})$ & - & 15 & 1.9 \\
\hline Case 8 & $\operatorname{cbl} A$ & $16(\mathrm{AF}, \mathrm{U})$ & - & 34 & 2.4 \\
\hline Case 9 & $\operatorname{cbl} A$ & $21(\mathrm{U})$ & - & N.D. & 1.0 \\
\hline Case 10 & $m u t$ & $35(\mathrm{U})$ & - & N.D. & 1.8 \\
\hline Case 11 & $\operatorname{cbl} A$ & $20(U)$ & - & N.D. & 1.4 \\
\hline Case 12 & $c b l A$ & $23(U)$ & + & N.D. & 6.1 \\
\hline
\end{tabular}

${ }^{1}$ The mutant class of each proband was determined by complementation analysis (19).

${ }^{2}$ Fluids studied were amniotic fluid (AF) and/or maternal urine (U).

${ }^{3}$ Disease status of the fetus was determined by propionate fixation of amniotic fluid cells, with (+) designating an affected fetus and (-) designating an unaffected fetus.

${ }^{4}$ N.D. $=$ not determined.

${ }^{5}$ Mean \pm 1 S.D.

\section{MATERIALS AND METHODS}

Chemical materials. Malonic acid diethyl ester and trideuteroidomethane (>99\% enriched) used in the synthesis of deuteriumlabeled MMA were purchased from Sigma (St. Louis, MO). Ethyl acetate (Gold Label) obtained from Aldrich Chemical Co. (Milwaukee, WI) was found to be of sufficient quality for organic acid extraction, whereas several other sources of ethyl acetate tested were of variable quality and often contained substances which interfered with mass spectral analysis. Diazomethane was generated from $N$-nitrosomethylurea purchased from K\&K Rare and Fine Chemicals (Plainview, NY). Gas chromatography was performed using coiled glass columns $(2 \mathrm{~mm} \times 6 \mathrm{ft})$ obtained from Supelco, Inc. (Bellefonte, PA) packed with 5\% SE-30 coated in our laboratory on silanized Anakrom (80-90 mesh) from Analabs (North Haven, CT). All other chemicals were reagent grade and were obtained from standard commercial sources.

Patient materials. Twelve pregnancies at-risk for carrying a fetus with methylmalonic acidemia were studied. These pregnant women previously had an affected child. The gestational ages at which each of these pregnancies were studied, the biologic fluids examined for each pregnancy (amniotic fluid and/or maternal urine), and the complementation class for which each fetus was at-risk are listed in Table 1. A control group of fourteen women undergoing amniocentesis between the 15-24 weeks of gestation for unrelated reasons was also studied. Gestational age was determined by sonographic measurement of fetal biparietal diameter. Amniotic fluids were obtained by standard techniques of transabdominal percutaneous puncture during the second trimester. Amniotic fluid cells were separated by centrifugation, placed in culture, and analyzed for $\left[1-{ }^{14} \mathrm{C}\right]$ propionate fixation (18). Amniotic fluid supernatants and urine samples obtained at the time of amniocentesis were stored at $-20^{\circ} \mathrm{C}$ until analysis.

Principles of analysis. The principles of the stable isotope dilution method for the measurement of low concentrations of biomolecules have been reviewed recently (4). In brief, a stable isotopically labeled analogue of the biomolecule is prepared and added to the biologic fluid to be studied. It is then carried through all stages of sample preparation and analyzed by on-line GC-MS. The analogue serves two functions: (1) it serves as a carrier for the minute amounts of naturally occurring (unlabeled) compound through all stages of analysis and (2) it serves as an internal standard for quantitation. The labeled and unlabeled compounds co-elute from the GC, but generate different ions in the MS depending upon the number of stable isotopic atoms and their location in the labeled compound. When operated in the SIM mode, the on-line GC-MS concurrently and selectively detects and quantitates the ions generated from the unlabeled and labeled compounds. The relative mass spectral response for corresponding mass fragments is determined by analysis of mixtures containing known amounts of the two compounds. The amount of unlabeled compound in an unknown sample is determined from these relative responses and from the amount of labeled compound added to the sample.

Synthesis of stable isotopically labeled MMA. A trideuteroanalogue of MMA, 2- $\left[{ }^{2} \mathrm{H}_{3}\right]$ methylmalonic acid, was synthesized via the malonate ester method $(3,22)$. The anion of malonate diethyl ester was prepared by adding freshly generated sodium ethoxide to malonate diethyl ester and alkylated with an equimolar amount of trideuteroiodomethane. The desired product, (2$\left[{ }^{2} \mathrm{H}_{3}\right]$ methyl)malonate diethyl ester, was separated from the dialkylated side-product, $\left(2,2-\left[{ }^{2} \mathrm{H}_{6}\right]\right.$-dimethyl $)$ malonate diethyl ester, by selective saponification of the former with $50 \%$ aqueous potassium hydroxide for $2 \mathrm{~h}$. The bulk of the 2,2-dimethylmalonate diethyl ester remained unsaponified under these conditions and was removed from the saponified compounds by ethyl ether extraction. The monomethylated product, which was quantitatively saponified under these conditions and remained in the water phase as the potassium salt, was approximately $20 \%$ contaminated with the dimethylated product. It was purified further as the free acid by repetitive fractional crystallization (3-4 times) from ethyl ether/hexane $(1: 3)$. The final crystalline $\left(2-\left[{ }^{2} \mathrm{H}_{3}\right]\right.$ methyl)malonic acid (D DMA) was -M9\% chemically pure and had an isotope enrichment greater than $99 \%$ (Fig. 1).

Preparation of biologic fluids for analysis. $\mathrm{D}_{3}$-MMA was added to the amniotic fluid supernatant $(0.5-3.0 \mathrm{ml})$ at a final concentration of $1 \mu \mathrm{g} / \mathrm{ml}$. The sample was deproteinized by acidification with $6 \mathrm{~N} \mathrm{HCl}$ to $\mathrm{pH} 1$ and was saturated with an excess of $\mathrm{NaCl}$. After centrifugation to remove the precipitates, the sample was resaturated with an excess of sodium chloride, extracted four times with an equal volume of ethyl acetate, dried over anhydrous sodium sulfate, and methylated with gaseous diazomethane, as previously described (14). The methylated sample was reduced to a small volume (50-200 $\mu \mathrm{l})$ under a gentle stream of nitrogen gas at room temperature. Samples were stable almost indefinitely when sealed carefully and stored at $4^{\circ} \mathrm{C}$. Urine samples (1.0-3.0 
$\mathrm{ml}$ ) were prepared in the same way except that deproteinization was unnecessary. MMA concentrations in amniotic fluid and urine were expressed in $\mathrm{ng} / \mathrm{ml}$ and $\mu \mathrm{g} / \mathrm{mg}$ creatinine, respectively, with creatinine concentrations determined as described by Taussky (17).

GC-MS studies. Aliquots of the methanolic solution of MMA dimethyl ester $(2-10 \mu \mathrm{l})$ were analyzed on a $5 \%$ SE-30 glass column in a Hewlett-Packard 5985A GC-MS equipped with a MX-E computer. Helium was used as the carrier gas for electron impact (EI) analysis at a flow rate of $25 \mathrm{ml} / \mathrm{min}$, whereas methane was used as both carrier gas at a flow rate of $10 \mathrm{ml} / \mathrm{min}$ and reactant gas $\left(2 \times 10^{-4}\right.$ Torr $)$ for chemical ionization (CI) analysis. The injection port temperature, the transfer line temper ture, and the ion source temperature were maintained at $225^{\circ} \mathrm{C}, 200^{\circ} \mathrm{C}$, and $200^{\circ} \mathrm{C}$, respectively. The ionizing voltage was $70 \mathrm{eV}$. The column temperature was initially $80^{\circ} \mathrm{C}$ and was increased at a rate of $6^{\circ} \mathrm{C} / \mathrm{min}$ following an initial delay of $0.5 \mathrm{~min}$. Under these conditions MMA dimethyl ester eluted at approximately $5 \mathrm{~min}$ after injection. A scan delay of $3 \mathrm{~min}$ was used to avoid the solvent peak. In both EI and CI studies, a mass range of 40-450 amu was scanned at a rate of $300 \mathrm{amu} / \mathrm{sec}$ in the acquisition mode. In the SIM mode, four ions (i.e., two corresponding ion pairs) were repetitively scanned with a $50 \mathrm{msec}$ dwell time/ion. Areas of the SIM peaks were integrated under computer control.

\section{RESULTS}

Determination of mass spectral conditions. The EI fragmentation patterns of MMA and $D_{3}$-MMA were unsatisfactory for the SIM analysis of these compounds in amniotic fluid and urine because of a paucity of unique higher mass ions caused by extensive<smiles>CC(=O)C(C)C(C)=O</smiles><smiles>CC(=O)C(C(C)=O)C(C)(C)C</smiles>

$M W=146$

$M W=149$

Fig. 1. Structure of (A) methylmalonic acid and (B) $\left(2-\left[{ }^{2} \mathrm{H}_{3}\right]\right.$ methyl)malonic acid as their dimethyl esters. MW stands for molecular weight in daltons. fragmentation. CI, on the other hand, is a "milder" form of ionization and provided an adequate mass spectral pattern containing a sufficient abundance of protonated molecular ions and other higher mass ions. It was, therefore, used for all the experiments described below. Two major and two minor ions were produced for both MMA (Fig. 2A) and $\mathrm{D}_{3}$-MMA (Fig. 2B). The base peaks at m/z 115 (MMA) and m/z $118\left(\mathrm{D}_{3}-\mathrm{MMA}\right)$ are due to the $[\mathrm{M}-31]^{+}$ions formed by the loss of one methoxy radical from the dimethyl esters. The other major peaks at $\mathrm{m} / \mathrm{z} 147$ and $\mathrm{m} / \mathrm{z} 150$ for MMA and $\mathrm{D}_{3}$-MMA, respectively, represent the $[\mathrm{M}+1]^{+}$or protonated molecular ions. Two minor peaks, $\mathrm{m} / \mathrm{z}$ 175 and $\mathrm{m} / \mathrm{z} 187$ for MMA and m/z 178 and 190 for $\mathrm{D}_{3}$-MMA, are due to the $[\mathrm{M}+29]^{+}$and $[\mathrm{M}+41]^{+}$cluster ions, respectively. The $[\mathrm{M}+29]^{+}$and $[\mathrm{M}+41]^{+}$cluster ions are formed by reaction of the low abundance $\mathrm{C}_{2} \mathrm{H}_{5}{ }^{+}$and $\mathrm{C}_{3} \mathrm{H}_{5}{ }^{+}$ions present in the methane plasma and the intact molecule.

Determination of standard curves. Standard curves for the stable isotope dilution technique were developed in the following way. Standard solutions which contained a fixed amount of $\mathrm{D}_{3}$-MMA $(1 \mu \mathrm{g} / \mathrm{ml})$ and a variable amount of MMA $(0.005-10 \mu \mathrm{g} / \mathrm{ml})$ were prepared and carried through the procedure of extraction, derivatization, and analysis outlined above. The areas of the four principle ions formed from MMA and $\mathrm{D}_{3}$-MMA were determined and the ratio of the areas for the corresponding ion pairs (e.g., $\mathrm{m} / \mathrm{z} 147$ and $\mathrm{m} / \mathrm{z} 150$ ) were plotted as a function of the ratio of the amounts of MMA and $\mathrm{D}_{3}-\mathrm{MMA}$ in the sample. The plot for the corresponding $[\mathrm{M}+1]^{+}$ion pair $(\mathrm{m} / \mathrm{z} 147,150)$ is shown in Figure 3. There was a linear relationship over three orders of magnitude between the ratio of peak areas of the MMA-derived $(\mathrm{m} / \mathrm{z} 147)$ and the $\mathrm{D}_{3}$-MMA-derived $(\mathrm{m} / \mathrm{z}$ 150) ions and the ratio of the amounts of MMA and $\mathrm{D}_{3}$-MMA (correlation coefficient greater than 0.9999); however, day-to-day variation was significant despite efforts to keep all GC-MS parameters constant and we found that preparation of a standard curve concurrently with analysis of unknowns was necessary. Linear regression analysis was applied to each set of standards and was used to calculate the concentration of MMA in unknowns analyzed on the same day. Similar standard curves were determined for the three other ion pairs (data not shown).

The ratio of the amounts of MMA to $D_{3}-\mathrm{MMA}$ at which the standard curve deviates from linearity represents the sensitivity limit of the assay. Inspection of Figure 3 indicates that the lowest ratio limit for the $[\mathrm{M}+1]^{+}$ion pair is approximately 0.01 , corresponding to an MMA concentration of $10 \mathrm{ng} / \mathrm{ml}$. Standard plots for the less abundant fragment clusters show deviation at the



Fig. 2. Chemical ionization (CI) mass spectra of methylmalonic acid (MMA) and (2- $\left[{ }^{2} \mathrm{H}_{3}\right]$ methyl)malonic acid (D $\left.-\mathrm{MMA}\right)$ dimethyl esters. The ions to which the various peaks correspond are discussed in the text. A 10 -fold decrease in the ordinate scale (\% relative abundance) for the higher mass ions is indicated by and to the right of the dotted line. 


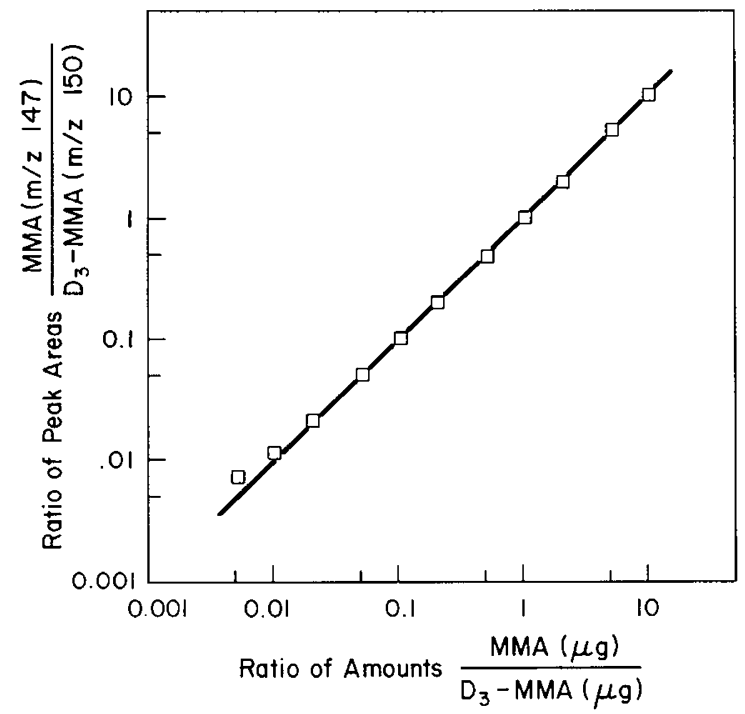

Fig. 3. Relationship between the ratio of amounts of methylmalonic acid (MMA) and $\left(2-\left[{ }^{2} \mathrm{H}_{3}\right]\right.$ methyl)malonic acid $\left(\mathrm{D}_{3}-\mathrm{MMA}\right)$ and the ratio of peak areas of the MMA-derived ion (m/z 147) and the $\mathrm{D}_{3}$-MMA-derived iọn $(\mathrm{m} / \mathrm{z} 150)$. This plot was obtained by analyzing a series of standard solutions of MMA and $\mathrm{D}_{3}$-MMA as described in the text.

slightly greater ratio of 0.02 . These sensitivity limits are approximately 2-4-fold lower than the observed concentration of MMA in amniotic fluid from normal women (see data below). Thus, this method is sufficiently sensitive to measure accurately the MMA concentration of such fluids.

Selection of SIM conditions for biologic fluids. Each of the four ion pairs was evaluated for suitability for analysis of MMA in amniotic fluid and urine. The $\mathrm{m} / \mathrm{z} 115,118$ pair was unsuitable because co-eluting compounds other than MMA or $\mathrm{D}_{3}$-MMA produced ions possessing the same $\mathrm{m} / \mathrm{z}$ values. The SIM plots for the $\mathrm{m} / \mathrm{z} 175,178$ ion pair were generally free of interference from partially co-eluting compounds but were unsuitable because they were often present at too low a relative abundance for reproducible quantitation. The remaining ion pairs, $\mathrm{m} / \mathrm{z} 147,150$ and $\mathrm{m} / \mathrm{z} 187$, 190 , were generally satisfactory for analysis. The $\mathrm{m} / \mathrm{z} 147,150$ ion pair was unusable on a few occasions because interfering substances were present, whereas the $\mathrm{m} / \mathrm{z} 187,190$ ion pair was occasionally unsatisfactory because its relative abundance was too low for reproducible quantitation. We found that information on both ion pairs were complementary and therefore we routinely monitored both of them. In no instance were both pairs unsatisfactory. MMA concentrations were calculated for each ion. Agreement between the values calculated for each ion pair generally agreed within $10 \%$, with averages calculated for a series of samples performed on the same day agreeing within 7\%. In approximately $95 \%$ of the samples the $\mathrm{m} / \mathrm{z} 147,150$ ion pair proved satisfactory and the results cited below were based on this ion pair alone. In the remaining $5 \%$ of cases, results cited were based on the $\mathrm{m} / \mathrm{z}$ 187,190 ion pair.

Similar results were found when each of the four ion pairs was evaluated for suitability for analysis of MMA in maternal urine. Again the $\mathrm{m} / \mathrm{z} 147,150$ ion pair was selected as the primary source for calculating the MMA concentration, while the $\mathrm{m} / \mathrm{z} 187,190$ ion pair served as an alternate.

Recovery and precision of the assay. The recovery and precision of the stable isotope dilution assay were evaluated in the following way. Amniotic fluids from several normal women were pooled and divided into ten equal aliquots of $3 \mathrm{ml}$ each. Supplemental MMA (100 ng/ml) was added to five of these aliquots. All ten samples were carried through the routine procedure of extraction, derivatization, and analysis. Recovery was quantitative (106\%) and the precision was excellent (Table 2). The coefficient of variation for measurements of the concentration of MMA in normal amniotic fluid was $7 \%$, whereas determinations of the higher range of values for the supplemented samples had a coefficient of variation of $2 \%$.

Concentration of $M M A$ in amniotic fluid from normal and heterozygous women. The concentration of MMA in amniotic fluids from normal and heterozygous pregnant women are shown in Table 1. The mean concentration for normal women examined during the 15-24th weeks of gestation was $38 \pm 15 \mathrm{ng} / \mathrm{ml}$ (mean \pm 1 S.D.). No significant difference was found when the women were divided into early gestational ( $<18$ weeks) and late gestational ( $>18$ weeks) groups. These data differ from a previously reported estimate, which was approximately 20 -fold higher (14). Amniotic fluids from eight pregnant heterozygotes were also examined. In six pregnancies (Cases 2, 4-8) the amniotic fluid MMA concentration was within two standard deviations of the mean of controls. A typical SIM chromatogram of amniotic fluid from an unaffected fetus is shown in Figure 4. Amniotic fluid cells derived from these pregnancies exhibited normal levels of $\left[1-{ }^{14} \mathrm{C}\right]$ propionate fixation (96-180\% of controls). We do not know if the fetuses examined in these cases were homozygous normal or heterozygous because the propionate fixation assay does not distinguish between these cases (18). Cases 1 and 3, on the other hand, had dramatically elevated MMA concentrations of 4,840 and $10,600 \mathrm{ng} / \mathrm{ml}$, respectively, representing approximately 125 250 -fold elevations as compared to the control group. The SIM chromatogram from Case 1 is shown in Figure 5. Amniotic fluid cell studies exhibited decreased $\left[1-{ }^{14} \mathrm{C}\right]$ propionate fixation $(<6.5 \%$ of controls), indicating that both fetuses were affected. The pregnancy carrying fetus 1 was aborted subsequently and the diagnosis of methylmalonic acidemia confirmed by direct enzyme analysis of fetal liver tissue ( $<1 \%$ mutase activity). The diagnosis for fetus 3 was confirmed by propionate fixation analysis of cultured amniotic fluid cells obtained at termination of pregnancy. There was concordance between the amniotic fluid cell studies and the amniotic fluid MMA studies for the six unaffected and two affected fetuses studied.

Concentration of $M M A$ in urine from normal and heterozygous women. The MMA concentration in urines from normal and heterozygous pregnant women is shown in Table 1 . The mean

Table 2. Recovery of methylmalonic acid from amniotic fluid

\begin{tabular}{cccc}
\hline $\begin{array}{c}\text { Amount added } \\
(\mathrm{ng} / \mathrm{ml})\end{array}$ & $\begin{array}{c}\text { Amount detected } \\
(\mathrm{ng} / \mathrm{ml})\end{array}$ & $\begin{array}{c}\text { Recovery } \\
(\%)\end{array}$ & $\begin{array}{c}\text { Number of } \\
\text { experiments }\end{array}$ \\
\hline 0 & $43 \pm 3^{1}$ & & 5 \\
100 & $149 \pm 3$ & 106 & 5 \\
\hline
\end{tabular}

${ }^{1}$ Values given as mean \pm 1 S.D.

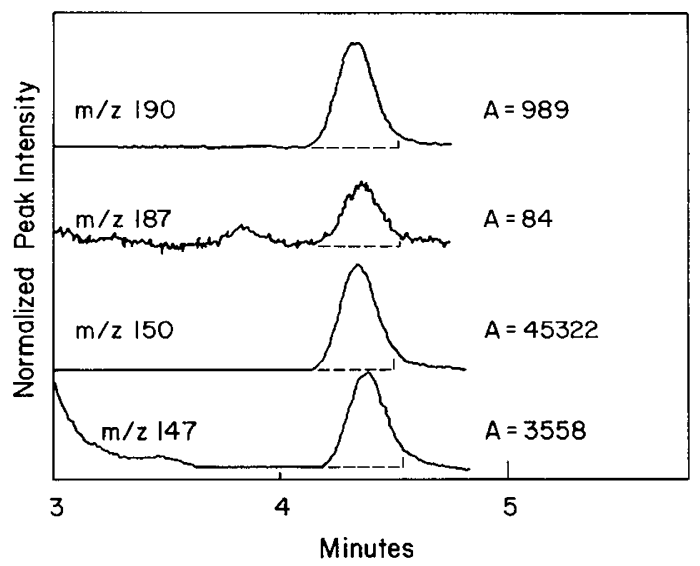

Fig. 4. Selected ion monitoring (SIM) chromatograms of amniotic fluid from a pregnant heterozygote carrying an unaffected fetus (Case 5). Four ions (m/z 147, 150, 187, and 190) were monitored with a $50 \mathrm{msec}$ dwell time per ion. Computer-controlled integrations for each peak area (A) are indicated to the right of each SIM chromatogram. The areas integrated are indicated by the dotted lines. 


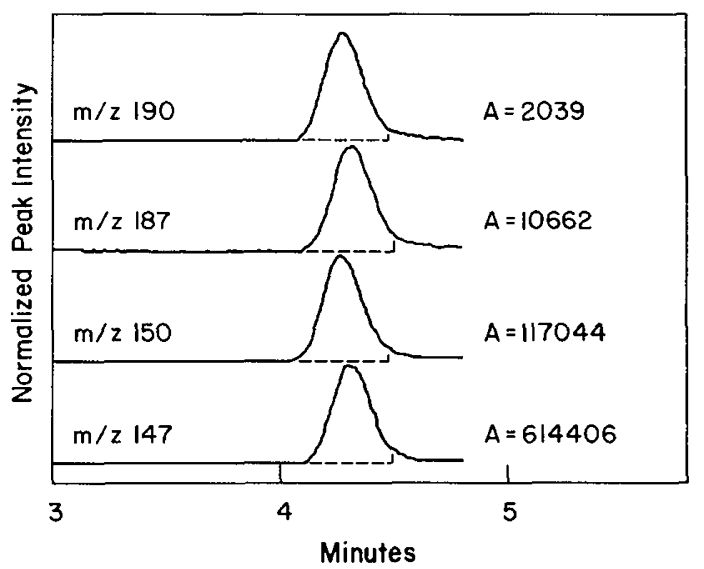

Fig. 5. Selected ion monitoring (SIM) chromatograms of amniotic fluid from a pregnant heterozygote carrying an affected fetus (Case 1). See the legend to Figure 4 for details.

concentration for normal women examined during the 15 th to the 24th week of gestation was $1.7 \pm 0.5 \mu \mathrm{g} / \mathrm{mg}$ creatinine (mean \pm 1 S.D.), which agrees well with previous estimates (14). Urine from ten pregnant heterozygotes was also examined. In eight of these pregnancies (Cases 4-11), the urinary MMA concentration was within two standard deviations from the mean of controls. Amniotic fluid cell studies demonstrated normal propionate fixation in these eight pregnancies. In contrast, the remaining two cases (Cases 3 and 12) exhibited decreased propionate fixation and elevated urinary MMA concentration (15.5 and $6.1 \mu \mathrm{g} / \mathrm{mg}$ creatinine, respectively), representing approximately 31/2-9-fold elevations as compared to the control group. Unfortunately, amniotic fluid for MMA determination was not available from Case 12 for the present study. There was concordance between the amniotic fluid cell studies and maternal urinary MMA concentrations for the eight unaffected and two affected fetuses studied.

\section{DISCUSSION}

We have developed a highly sensitive, specific, and reproducible method for the measurement of methylmalonic acid in amniotic fluid and urine, and have demonstrated its usefulness for the rapid and reliable prenatal diagnosis of methylmalonic acidemia. This new method affords significant advantages over other currently used approaches for prenatal diagnosis of this disease.

The concentrations of MMA is normally very low in the body fluids of healthy individuals, including amniotic fluid in pregnant women. Currently used methods for the measurement of MMA, including colorimetry, gas chromatography, and liquid chromatography, are not sufficiently sensitive to measure accurately the low MMA concentration in normal amniotic fluid. We previously reported a micromethod for the measurement of MMA in amniotic fluid, urine, and serum based on the prefractionation of organic acids by anion-exchange chromatography followed by gas chromatography, and its application to the prenatal diagnosis of methylmalonic acidemia (14). We were concerned that our estimates of MMA concentration in normal amniotic fluid by this previous method were inflated by a co-eluting contaminant. Similar problems exist for currently used liquid chromatography (LC) methods of analysis. Microanalysis by either GC or LC alone has three inherent limitations: (1) the limited resolving capability of the instrument; (2) the relative non-specificity of the detection systems; and (3) the significant adsorptive effects of the column support materials. Limitations (1) and (2) can be at least partially alleviated by utilizing an on-line GC-MS system operated in the SIM mode, wherein key ions may be used for detection, resolution, and quantitation of co-eluting substances. SIM-GC-MS will not circumvent limitation (3), however, because the errors introduced by the GC column support precede the introduction of the sample into the MS. The stable isotope dilution technique circumvents this limitation by providing a stable isotopically-labeled analogue of MMA $\left(D_{3}\right.$-MMA) as a carrier, which together with the natural compound, saturates the adsorptive sites of the column support. The value determined in this study for the concentration of MMA in amniotic fluid from normal pregnant women is 20 -fold less than our previous estimate (14), thereby confirming our suggestion that co-eluting material was falsely elevating the MMA determination for amniotic fluid. Elimination of interference by this coeluting material also amplifies the difference observed between MMA levels in pregnancies carrying an affected fetus from those carrying an unaffected fetus. Affected fetuses in Cases 1 and 3 produced amniotic fluid MMA concentration elevations greater than two orders of magnitude compared to controls.

We have identified three affected fetuses by the described method: one by amniotic fluid MMA determination alone (Case 1), one by urinary MMA determination alone (Case 12), and one by both amniotic fluid and urinary MMA determinations (Case 3). Concordance was observed in six at-risk pregnancies between amniotic fluid cell studies (propionate fixation), amniotic fluid MMA levels, and urinary MMA levels. Assuming that the amniotic fluid MMA level determined for Case 12 was at least qualitatively correct as previously reported $(2.1 \mu \mathrm{g} / \mathrm{ml}$ at 19 week gestation, see reference 14), Case 12 was also concordant for these three analytical methods. The remaining five cases were concordant for cell studies and either amniotic or urinary MMA levels, depending upon which was performed. These three analytical methods are complementary. We recommend that prenatal diagnosis of methylmalonic acidemia should be based whenever possible on both amniotic fluid cell studies and amniotic fluid MMA measurement as described in this report. If this is not possible we propose that either of these methods is sufficiently reliable to be used alone.

The data on urinary MMA excretion are as yet not sufficiently convincing to permit counseling based on the results of that test alone. Affected fetuses produced relatively smaller elevations in urinary MMA excretion (31/2-9-fold) compared to the elevations observed in amniotic fluid MMA concentrations from pregnancies carrying affected fetuses (125-250-fold), and one of the urines (Case 12) was not obtained until 23 weeks gestation. Nevertheless, these urinary elevations are significant (9-28 standard deviations above the mean), and it remains to be seen if similar elevations in urinary MMA excretion will be observed in other at-risk pregnancies. Presently we do not advocate the use of urinary MMA excretion as the sole determinant for prenatal diagnosis. Urinary MMA excretion is suitable, however, for monitoring the effects of prenatal vitamin $B_{12}$ therapy of affected fetuses (1).

Several questions remain unanswered regarding the prenatal diagnosis of methylmalonic acidemia using the present method. First, how early in pregnancy does the elevation in amniotic fluid MMA level become manifest? The findings in Case 3 suggest that amniotic fluid MMA levels are significantly elevated by 16 weeks gestation. The studies in Cases 1 and 12 were performed slightly later in pregnancy (18 and 19 weeks, respectively) but also within the period in which the results are usable for further pregnancy planning. Second, do all complementation classes exhibit elevations in amniotic fluid MMA concentrations? Cases 1, 3 and 12 belong to the $c b l B$, mut, and $c b l A$ complementation groups, respectively. It is not known whether an affected fetus of the $c b l$ $C$ or $c b l D$ complementation class would also produce elevated MMA levels. Postnatally, some of these individuals have exhibited milder elevations of serum MMA and urinary MMA excretion than do those of the other three complementation groups $(6,8,9)$. No affected fetus from either the $c b l C$ or $c b l D$ group has been diagnosed prenatally by any method. The highly sensitive assay described in this report may be essential for the prenatal diagnosis of these disorders using amniotic fluid if there is a less dramatic elevation of amniotic fluid MMA in the early midtrimester of pregnancy.

During the course of these studies, Naylor et al. (15) reported a method for measurement of methylcitric acid by stable isotope 
dilution GC-MS and its successful application to the prenatal diagnosis of propionic acidemia and methylmalonic acidemia. There are important differences between the method which they describe and our own. First, our method has two technical advantages: (1) the synthesis of $\mathrm{D}_{3}$-MMA is considerably easier than synthesis of the deuterated analogue of methylcitric acid described by Naylor et al:; and (2) the liquid partition chromatography system employed by Naylor et al. is not available in most laboratories whereas the liquid-liquid extraction technique we employed requires no special equipment. Second, in a physiologic sense, a diagnostic method for methylmalonic acidemia based on measurement of methylmalonic acid is intrinsically more direct and sensitive than a method based on analysis of methylcitric acid. Naylor et al. reported 5-7-fold elevations in the concentration of methylcitric acid in amniotic fluids from pregnancies carrying fetuses affected with methylmalonic acidemia compared to controls. Our results indicate 125-250-fold elevations in the concentration of MMA in pregnancies carrying affected fetuses. Thus, the distinction between pregnancies carrying an affected and an unaffected fetus is much more evident, and the diagnosis of an affected fetus more convincing, when based on the measurement of amniotic fluid MMA rather than methylcitric acid. On the other hand, the stable isotope dilution analysis of methylcitric acid is of more general use than our method because it can be used for prenatal diagnosis of propionic acidemia and multiple carboxylase deficiency, as well as methylmalonic acidemia.

In conclusion, we have developed a stable isotope dilution method for measurement of MMA that has permitted the first accurate determination of the concentration of MMA in amniotic fluid from healthy, pregnant women. More importantly, the method provides a reliable technique for prenatal diagnosis of methylmalonic acidemia that is complementary to amniotic fluid cell studies, and may supplant such studies in selected instances. The stable isotope dilution method can be applied to other inborn errors of metabolism, many of which are not currently diagnosable by other approaches. We are presently extending this method to such disorders.

\section{REFERENCES AND NOTES}

1. Ampola, M. G., Mahoney, M. J., Nakamura, E., and Tanaka, K.: Prenatal therapy of a patient with vitamin $\mathrm{B}_{12}$-responsive methylmalonic acidemia. N. Engl. J. Med., 293: 313 (1975).

2. Bakker, H. D., Van Gennip, A. H., Duran, M., and Wadman, S. K.: Methylmalonate excretion in a pregnancy at risk for methylmalonic acidaemia. Clin. Chim. Acta, 86: 349 (1978)

3. Baretz, B. H., Lollo, C. P., and Tanaka, K.: Synthesis of short chain carboxylic acids labelled with ${ }^{13} \mathrm{C}$ and ${ }^{2} \mathrm{H}$ at various positions. J. Labelled Comp., 15:369 (I978).

4. Bjorkhem, I.: A review on selective ion monitoring in clinical chemistry. Critical Revs. Clin. Lab. Sci., 11: 53 (1979).

5. Buchanan, P. D., Kahler, S. G., Sweetman, L., and Nyhan, W. L.: Pitfalls in the prenatal diagnosis of propionic acidemia. Clin. Genet., 18: 177 (1980).

6. Dillon, M. J., England, J. M., Gompertz, D., Goodey, P. A., Grant, D. B., Hussein, H. A.-A., Linnell, J. C., Mathews, D. M., Mudd, S. H., Newns, G. H., Seakins, J. W. T., Uhlendorf, B. W., and Wise, I. J.: Mental retardation, megaloblastic anaemia, methylmalonic aciduria and abnormal homocysteine metabolism due to an error in vitamin $B_{12}$ metabolism. Clin. Sci. Mol. Med., 47: 43 (1974).

7. Gompertz, D., Goodey, P. A., Saudubray, J. M., Charpentier, C., Chignolle, A., and Girard, S.: Prenatal diagnosis of methylmalonic aciduria. Pediatrics, 54: 511 (1974).

8. Goodman, S. I. Moe, P. G., Hammond, K. B., Mudd, S. H., and Uhlendorf, B. W.: Homocystinuria with methylmalonic aciduria: two cases in a sibship. Biochem. Med., 4: 500 (1970).

9. Levy, H. L., Mudd, S. H., Schulman, J. D., Dreyfus, P. M., and Abeles, R. H.: A derangement in $B_{12}$ metabolism associated with homocystinemia, cystathioninemia, hypomethioninemia and methylmalonic aciduria. Am. J. Med., 48: 390 (1970).

10. Mahoney, M. J., Rosenberg, L. E., Lindblad, B., Waldenstrom, J., and Zetterstrom, R.: Prenatal diagnosis of methylmalonic aciduria. Acta Pediatr. Scand. 64: 44 (1975).

11. Matsui, S. M., Mahoney, M. J., and Rosenberg, L. E.: The natural history of the inherited methylmalonic acidemias. Am. J. Hum. Genet., 32: 119A (1980).

12. Morrow, G. III, Schwarz, R. H., Hallock, J. A., and Barness, L. A.: Prenatal detection of methylmalonic acidemia. J. Pediatr., 77: 120 (1970)

13. Morrow, G. III, Revsin, B., Mathews, C., and Giles, H.: A simple, rapid method for prenatal detection of defects in propionate metabolism. Clin. Genet., 10: 218 (1976)

14. Nakamura, E., Rosenberg, L. E., and Tanaka, K.: Microdetermination of methylmalonic acid and other short chain dicarboxylic acids by gas chromatography: use in prenatal diagnosis of methylmalonic acidemia and in studies of isovaleric acidemia. Clin. Chim. Acta, 68: 127 (1976).

15. Naylor, G., Sweetman, L., Nyhan, W. L., Hornbeck, C., Griffiths, J., Morch, L., and Brandange, S.: Isotope dilution analysis of methylcitric acid in amniotic fluid for the prenatal diagnosis of propionic and methylmalonic acidemia. Clin. Chim. Acta, 107: 175 (1980)

16. Rosenberg, L. E. and Scriver, C. R.: Disorders of amino acid metabolism. In: P K. Bondy, L. E. Rosenberg: Metabolic Control and Disease. Ed. 8, p. 583 (W. B. Saunders, Philadelphia, 1980)

17. Taussky, H.: A microcolorimetric determination of creatinine in urine by the Jaffe reaction. J. Biol. Chem., 208: 853 (1954).

18. Willard, H. F., Ambani, L. M., Hart, A. C., Mahoney, M. J., and Rosenberg, L. E.: Rapid prenatal and postnatal detection of inborn errors of propionate, methylmalonate, and cobalamin metabolism: a sensitive assay using cultured cells. Hum. Genet., 34: 277 (1976).

19. Willard, H. F., Mellman, I. S., and Rosenberg, L. E.: Genetic complementation among inherited deficiencies of methylmalonyl CoA mutase activity: evidence for a new class of human cobalamin mutant. Am. J. Hum. Genet., 30: 1 (1978).

20. A preliminary version of this work was submitted to The American Society of Human Genetics meeting, Dallas, 1981.

21. Requests for reprints should be addressed to: Kay Tanaka, M.D., Yale University School of Medicine, Department of Human Genetics, 333 Cedar Street, P.O. Box 3333, New Haven, Connecticut 06510 (USA).

22. We would like to thank Mr. Bruce Baretz for assistance with the synthesis of D $_{3}$-MMA, Drs. M. Ampola, M. Cowger, M. Golbus, D. Harris, M. Sandstrom, and $M$. Yoshino for contributing amniotic fluid and urine samples from their patients to this study, and Ms. Marilyn Feldman for help in preparation of the manuscript.

23. This work was supported by a grant from the National Institutes of Health (AM 17453). A.B.Z. was supported by a fellowship from the National Institutes of Health (GM 07439).

24. Received for publication September 15, 1981

25. Accepted for publication March 2, 1982. 